\title{
A COMPUTATIONAL FRAMEWORK FOR EXPLORING THE SOCIO-COGNITIVE FEATURES OF TEAMS AND THEIR INFLUENCE ON DESIGN OUTCOMES
}

\author{
Singh, Harshika (1); Cascini, Gaetano (1); Casakin, Hernan (2); Singh, Vishal (3) \\ 1: Politecnico di Milano; 2: Ariel University; 3: Aalto University
}

\begin{abstract}
The dynamics of design teams play a critical role in product development, mainly in the early phases of the process. This paper presents a conceptual framework of a computational model about how cognitive and social features of a design team affect the quality of the produced design outcomes. The framework is based on various cognitive and social theories grounded in literature. Agent-Based Modelling (ABM) is used as a tool to evaluate the impact of design process organization and team dynamics on the design outcome. The model describes key research parameters, including dependent, independent, and intermediates. The independent parameters include: duration of a session, number of times a session is repeated, design task and team characteristics such as size, structure, old and new members. Intermediates include: features of team members (experience, learning abilities, and importance in the team) and social influence. The dependent parameter is the task outcome, represented by creativity and accuracy. The paper aims at laying the computational foundations for validating the proposed model in the future.
\end{abstract}

Keywords: Collaborative design, Process modelling, Design management

\section{Contact:}

Singh, Harshika

Politecnico di Milano

Department of Mechanical Engineering

Italy

harshika.singh@polimi.it

Cite this article: Singh, H., Cascini, G., Casakin, H., Singh, V. (2019) 'A Computational Framework for Exploring the Socio-Cognitive Features of Teams and their Influence on Design Outcomes', in Proceedings of the 22nd International Conference on Engineering Design (ICED19), Delft, The Netherlands, 5-8 August 2019. DOI:10.1017/dsi.2019.3 


\section{INTRODUCTION}

One way to approach innovation is through co-design or collaborative design, characterized by different team members working together in a joint effort to produce creative solutions. However, codesigning is not always a smooth process. While several factors affect the co-design process, communication among team members is the most important one. Effective communication is crucial to produce successful projects (Badke-Schaub and Frankenberger, 1999), and poor or unsatisfactory communication can unnecessarily enlarge the number of interactions among team members, which consequently result in delays and waste resources.

In order to gain better insight into team communication, team coordination and the generation of creative outcomes, a bottom-up approach to study co-design at a micro and macro-level is necessary. In this context, 'micro' refers to the factors at the individual level that are mainly concerned with the personal and cognitive characteristics (e.g., experience, domain knowledge, self-efficacy are some of them). 'Macro' on the other hand refers to the characteristics at project level (e.g., task type, team size, team influence, duration of the task, team structure). The individual characteristics help in learning how they give rise to the social influence on task outcome and team dynamics. Understanding of project characteristics is important as any changes in them have consequences on the individuals' ability to coordinate actions. Therefore, the development of a model capable of representing macro and micro-level characteristics would assist and improve the understanding about conducting efficient co-design activities. Ultimately, learning about how individual and project characteristics should be harmonised, would help to achieve better design outcomes. Several techniques facilitate fluent collaboration in co-design. Other approaches such as design thinking, agile, lean design, and waterfall address the problem at macro-level. However, these methods did not pay enough attention to smallscale interactions between individuals based on their differences to aid group dynamics in co-design. On the other hand, experimental observations and empirical analyses of team behaviour aim at providing insights at macro and micro-level. However, they are resource intensive, time consuming and, due to the variety of experimental parameters, they hardly support in predicting project outcomes and context variables.

In order to investigate how design outcomes are affected by micro-level characteristics of individuals that are influenced by the macro-level factors of a co-design system, the authors explore the use of Agent Based Modelling (ABM). ABM is useful in addressing autonomous and heterogeneous individual characteristics to represent and compute social dynamics of co-design activity. Computational tools are helpful in demonstrating the emerging phenomenon of a system based on input variables, rules and theories that guide the model (Abar et al., 2017).

In this paper the authors want to study how individual characteristics (learning by experience, attitude, forgetfulness and importance/role in the team) are influenced by project characteristics (task type, task duration, team structure, social influence and team size), with an eventual effect on the task outcome. The authors intent to use an Agent-Based Modelling (ABM) to describe the co-design system from the perspective of its agents' (individual) characteristics. The purpose of the work is to find out how agents (individuals) behave when they perform the same task over a period of time, while taking into account the individual, team and task characteristics, as well as their combined effect on the value (in terms of exploration of solution space and quality) of the solution. It is expected that the analysis of the co-design activities will help to gain a larger insight onto the interactions maintained by the agents during the process.

The paper starts with a literature review of the work done in the area of computational models for studying co-design activities, while identifying their limitations and gaps. Based on these, research questions that form the foundation of the model are presented. The next section describes the proposed conceptual model. This is followed by conclusion, limitations and future work for implementing the conceptual model.

\section{MODEL FOUNDATION}

Several studies used ABM as a tool for computational modelling and representing real-world scenarios of team collaboration. In the 1990s VDT (the Virtual Design Team model) was developed to simulate communication in engineering design teams based on the task types and design complexities (Jin and Levitt, 1996). However, VDT like other models that focused on organisation structure on project 
performance (Jamshidnezhad and Carley, 2015; Carley and Gasser, 1999) lacked the human behavioural aspect in their model. Other computational models interested in co-design from the perspective of human behaviour and teamwork focused on communication and collaboration issues. Singh and Casakin, (2015) and Singh (2009) investigated the effect of methods/tools on design team collaboration. Others explored team expertise, team experience on task performance (e.g., Gero and Kannengiesser, 2004; Perišić et al., 2019). Decision-making was examined by Sayama et al. (2010), Dionne and Dionne (2008), and Singh et al. (2011) by varying team structure and leadership type. Individual and team learning and their problem solving behaviour on design task were modelled by McComb et al. (2015), and Sosa and Gero (2013). Although previous works on computational modelling provide useful insights at micro-level elements of the co-design system, there are still plenty of issues to investigate: i) the existing models disregard the effect of time on the design outcome. For instance, how individual agent characteristics (e.g. expertise, experience, self-efficacy, motivation, or role/importance) affect the project outcome over project characteristics through time (e.g., number and duration of sessions); ii) There is a lack of studies onto how social issues related to the team influence idea generation and decision-making in co-design activity; iii) There is a need to learn more about how some agents become more important than others through team interactions, and how their role changes the decision making process. In short, not much attention was given to the cognitive and social features of a team working.

Starting from the above gaps and the scarce work done on the socio-cognitive aspects in literature, the objective of this work is to understand the effect of individual features (cognitive and behavioural) of agents over project characteristics, with consequences for the task output. In the proposed model, the focus on the task output is referred to the exploration of the solution space and the quality of the design solutions (see next section). Therefore, a conceptual framework to model these features is proposed.

The research goals and questions that guide this study can be grouped as: (1) Learning from experience, (2) Attitude towards work, (3) Conformity and (4) Role/importance.

1. Learning from experience:

a) What is the effect of previous experience on successive tasks outputs?

2. Attitude towards work:

a) How the self-efficacy of agent (individual) changes as experience is gained over time?

b) What is the effect of agent self-efficacy on task output?

3. Conformity (also team and social influence are used interchangeably):

a) What is the effect of conformity on decision-making?

i) When the same agents work together without replacing/adding new agents;

ii) When new agents are added to replace old ones.

4. Role/importance of some agents:

a) How some agents gain importance/influence others in a flat team (i.e. a team with no formal hierarchical relationships among the team members)?

b) How these agents affect idea generation and concept selection processes?

c) What is their effect on task output (exploration of solution space and quality)?

This paper proposes a conceptual model to address the above research questions building on the works mentioned hereafter.

There are few works in literature focusing on the learning abilities of agents deployed while performing a similar task. Therefore, the proposed model explores how agents experience influences the task outcome. Learning from experience is based on the power law of practice; success-based learning is taken into account when an agent learns differently due to success and failures of carrying out a task (Leibowitz et al., 2010). Real life situations are considered for this model, where every agent has different learning abilities. They learn differently from success and failures as well as they have different memory capacity, i.e. forgetting previous experience varies with each agent.

Self- efficacy refers to ones belief in the capacity to achieve goals. The model considers self-efficacy and motivation as the parameters for an agent's attitude towards work. These parameters are seen as critical for proposing solutions and making decision in group. Motivation and experience influences self-efficacy of an individual (Carberry et al., 2010). Self-efficacy also gives rise to influencing power, which influences team members and ultimately project outcomes (Prussia et al., 1998). Since there are no empirical studies supporting these, the present model will propose to explore this phenomenon and its cognitive effect on the task outcome. Figure 1, shows how self-efficacy helps in emergence of 
influencers for this model, which ultimately affect task outcomes. In this model, emergence of influencers is an important effect. As these influencers often influence other team members while generating and proposing solutions. While proposing solutions, individual team members might become influenced by the solutions suggested by these influencers, which they believe are better than their own one.

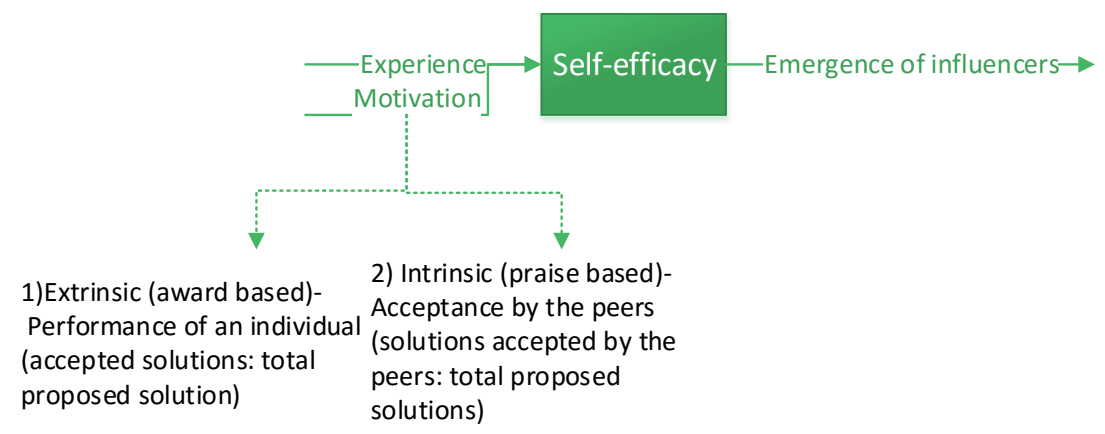

Figure 1. Factors giving rise to the emergence of influencers

'Conformity is the type of social influence where a person changes his/her attitude or behaviour in response to group pressure' (Sparks, 2018). Cambridge dictionary defines conformity as 'behaviour that follows the usual standards that are expected by a group or society'. In this model, conformity is considered in situations where agents take decision in accepting or rejecting solutions proposed by one team member. For the decision-making process, a specific solution is considered, and in order to be accepted by the group, an agent may change its public behaviour but not its personal beliefs (Kelman, 1958). Often, team members tend to consent with others in order to be accepted or to avoid disagreements, since this behaviour limits the exploration of the creative space. Hence, an aim of this model is to investigate the consequences of this social behaviour on the task output.

\section{MODEL DESCRIPTION}

The early design phase is modelled, as it is critical in new product development. At this phase, team members come up with solutions to address the problem proposed by the customer, or by the project manager. The task outcome is measured in terms of exploration of solution space and quality. This is because exploration of design space is one of the ways to measure/express creativity (Dorst and Cross, 2001). Design space could be briefly defined as a metaphorical context containing a set of possible alternatives describing design problems and design solutions to be developed. In this context, quality refers to the value of the solution (explained in section 3.3) in the design space proposed by any agent during the course of time. Based on the research questions, the model framework has the following qualities:

1. The model consists of agents (individual agents who form a team and a controller agent) (see Agents section).

2. An agent learns from its experience. Learning can change based on success or failure in proposing a solution.

3. An agent is influenced by conformity in decision-making and idea generation processes.

4. An agent's attitude (self-efficacy) changes with the level of expertise gained and motivation through the interactions with other agents.

5. Eventually, self-efficacy of an agent is considered to determine its importance in the team or, in other words, how influential is that agent.

Though the parameters used for modelling a team are not exhaustive, it can be said that still the proposed model largely captures the main aspects a co-design session. The model will be simulated using Python script.

\subsection{Parameters}

The selection of the parameters to represent in the agent-based model has been carried out based on the indications provided in (Salas et al., 2005) related to the components to be considered when simulating a team model. If one design session is zoomed in, many parameters play role in the task output. Figure 2 shows the parameters which are considered in the model. They are various input, 
control and resource parameters that affect the outcome. Based on the research questions, the parameters have been classified as independent, intermediate, and dependent.

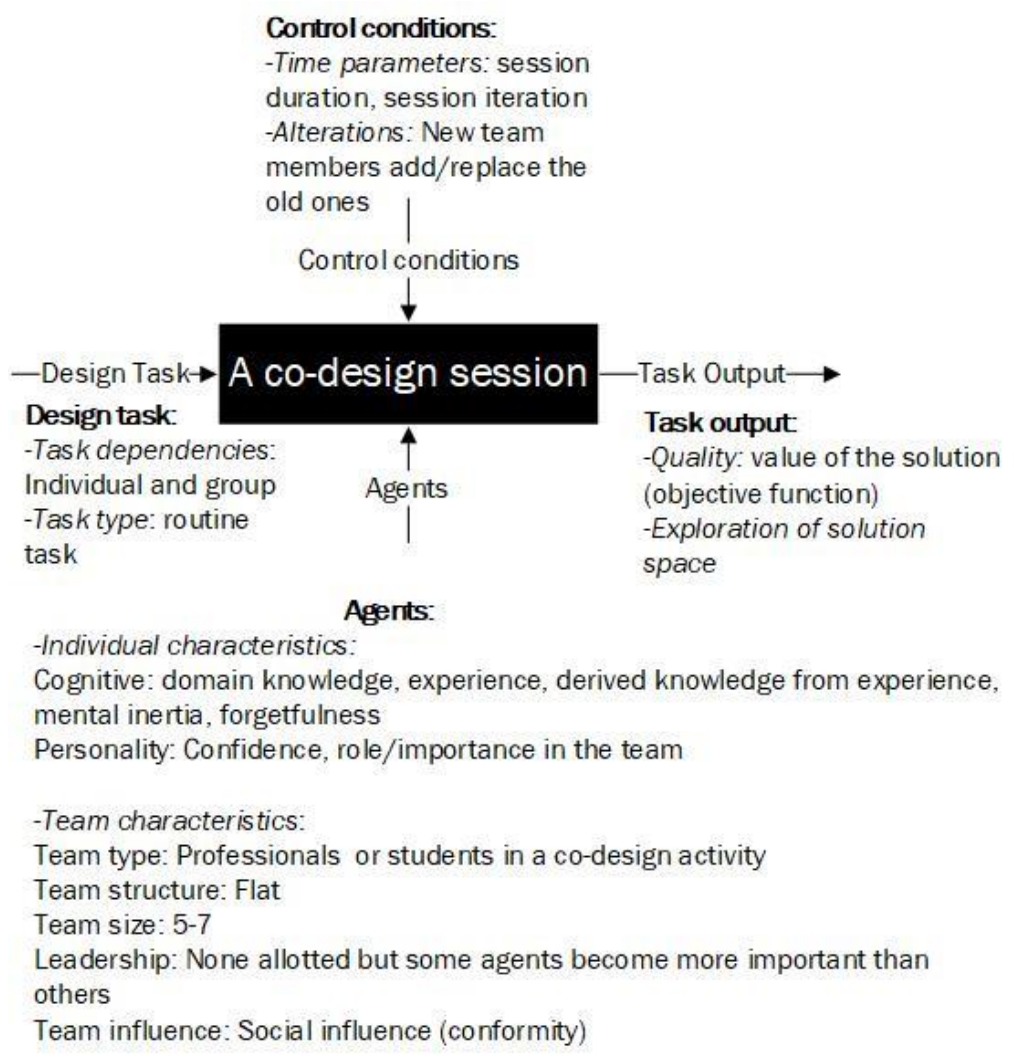

Figure 2. Parameters for the model

1. Independent parameters remain unaffected by the changes in the model occurring in control parameters like duration of a session, number of times a session is repeated, design task and team characteristics (type, size, number, structure, new members replacing old ones).

2. Intermediates are affected by the independent parameters, and in turn, affect the dependent parameters. These include features of individuals (forgetfulness, learning from experience and importance in the team) and social influence or conformity that changes with the independent parameters. Importance in the team or emergence as an influencer is in turn dependent on selfefficacy, which is effected by motivation and experience.

3. Dependent parameters are susceptible to changes in independent and intermediates. This includes output results in terms of exploration of solution space and quality of the solutions.

\subsection{Agents}

Agents for this work include:

1. Individual agents: Are individuals who form a team and work on the given task.

Their characteristics include:

a) Knowledge extraction: These agents (individual team members) search their given set of knowledge to propose solutions to the task. When proposing a solution, the agent can be influenced by its own experience or by what is proposed by the team.

b) Learning: As the agents perform the task, they learn from their experience.

c) Adapting: Agents are capable to act and adapt to social influence. They can be influenced with their own experience or the social information (conformity) to 'fit' in the team.

d) Decision making: Agents are capable of making their decision when it comes to choosing the solution proposed by the other team members. The probability that an agent will agree or disagree with the proposed solution will depend on the difference in distance between their 'preferred' solution and the proposed solution, quality as well as the social influence (conformity) of the team.

2. Controller agent: The control manager (in computational terms 'controller agent') is an individual who is responsible for: 
a) Assigning the task;

b) Adding and removing agents;

c) Providing feedback on whether or not the solution is acceptable;

d) Choosing the best solution the team came up in the allotted time.

The individual agents form a team where new agents (in real-world scenario where new members join the team or replace the old ones) can replace them. Flat team structure was considered in this model, which means that there is no middle management between employees and control managers. The idea behind this team structure is to represent more involved team members in the decision-making process. Flat team structures in real life scenarios can be seen in small organisations or start-ups. In many small and medium enterprises, the CEO or business owner is directly involved in the co-design process. Similarly, big organisations like Nike and Valve are adapting flat structure in their workforce to 'minimise bureaucracy and development times for new ideas'.

\subsection{Task}

Many approaches on agent's problem solving were investigated in large detail in the past (Herrmann, 2015). However, since the present model is at its initial phase of development, it still lacks many of such details. In this work, the task (or the design problem) is computationally represented as an objective function $\mathrm{f}(\mathrm{x})$, where the team has to maximise the quality of the design problem i.e. maximise the value of the objective function. Design problem solving (in this case doing a task) is based on the four basic underlying cognitive operations proposed by (Stempfle and Badke-Schaub, 2002) that include generation, exploration, comparison and selection. These are applied in various combinations while solving a problem as well as to collective process of teamwork. In the model, "process 2" is implemented as proposed by Stempfle and Badke-Schaub (2002) (see Figure 3).

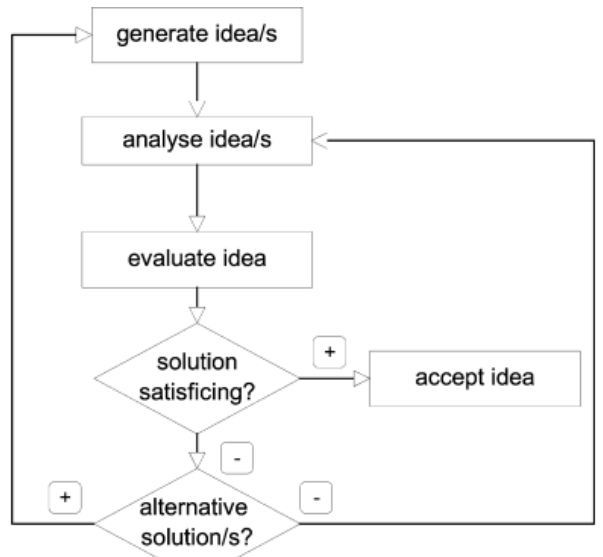

Figure 3. Process 2 that is implemented in the model (Stempfle and Badke-Schaub, 2002)

Based on Figure 3, in the given model an agent generates an idea, and analyses is based on its previous experience gained from on the ideas that the controller agent accepted or rejected in previous instances. A random agent is selected to propose an idea, while the other agents in the team evaluate it Thereafter, based on the idea they prefer or on social influence, they accept or reject it. When most of the team members agree with the idea, the solution is proposed to the controller agent. The controller agent checks whether the solution lies in the acceptable range or not, and accepts or rejects it accordingly. The key points of the model are the followings:

1. At the beginning, the information needed to do the task is randomly distributed among the agents.

2. None of the agents knows the complete solution space, but they know the boundary limits of the solution.

3. The controller agent knows the acceptable and unacceptable solution range.

4. The team consisting of ' $n$ ' individuals discusses a solution.

5. The design process is modelled as a sequence of iterative attempts, where agents propose solutions based on their previous learning, experience and social influence. The parameters vary over different length and number of sessions.

6. One iterative step consists of (see Figure 4):

a) The task is given by the controller agent; 
b) Individual agents start thinking about the solutions;

c) A speaker is randomly selected;

d) The speaker proposes a solution;

e) The other individual agents respond to the suggested solution;

f) Based on the team degree of agreement, the solution is passed to the controller agent;

g) The controller agent expresses his judgement about the proposed solution.

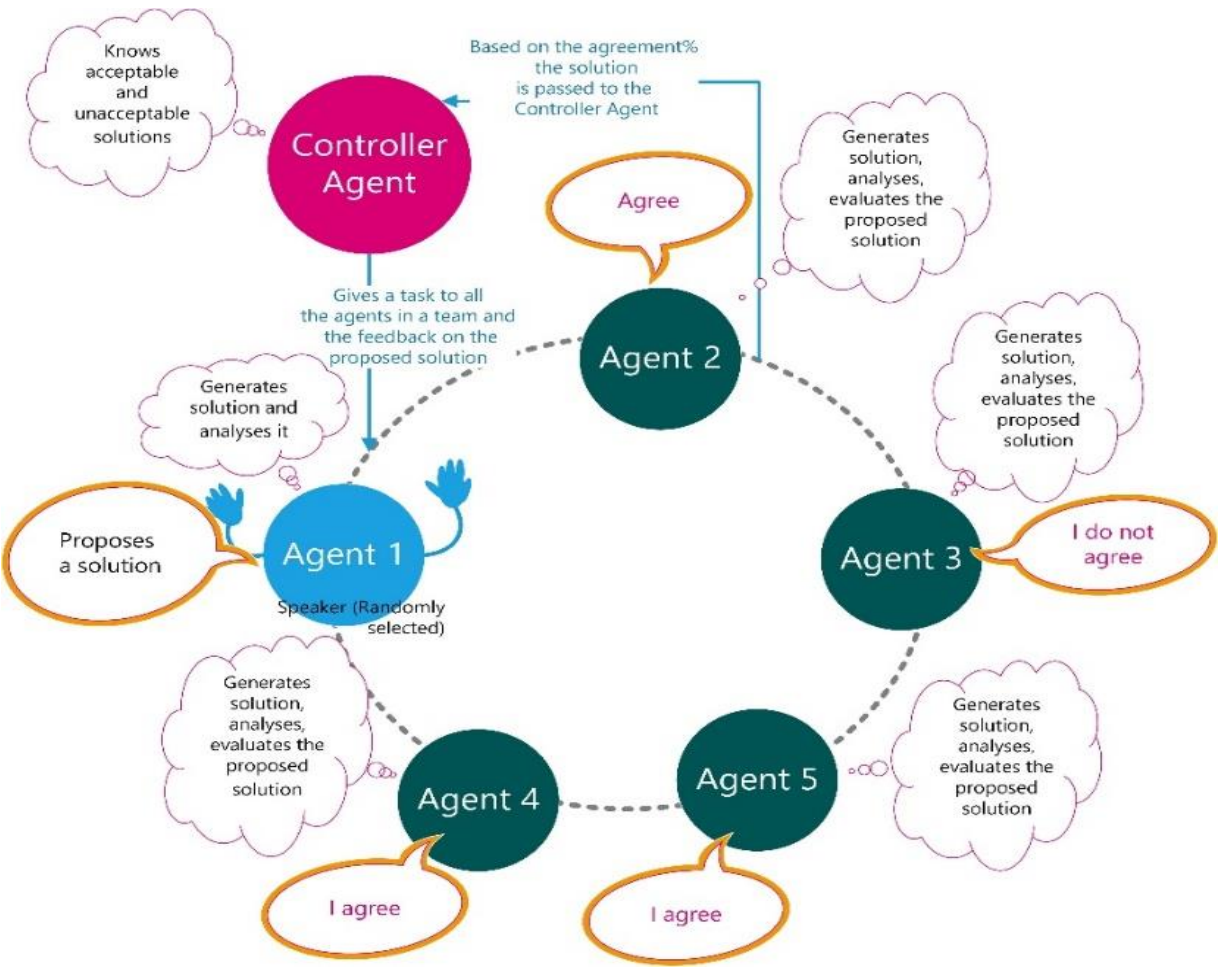

Figure 4. One iterative step

The design task given to the team may have more than one acceptable solution. The solution to the task may or may not be accepted by the controller agent (as it knows the range of acceptable solutions).

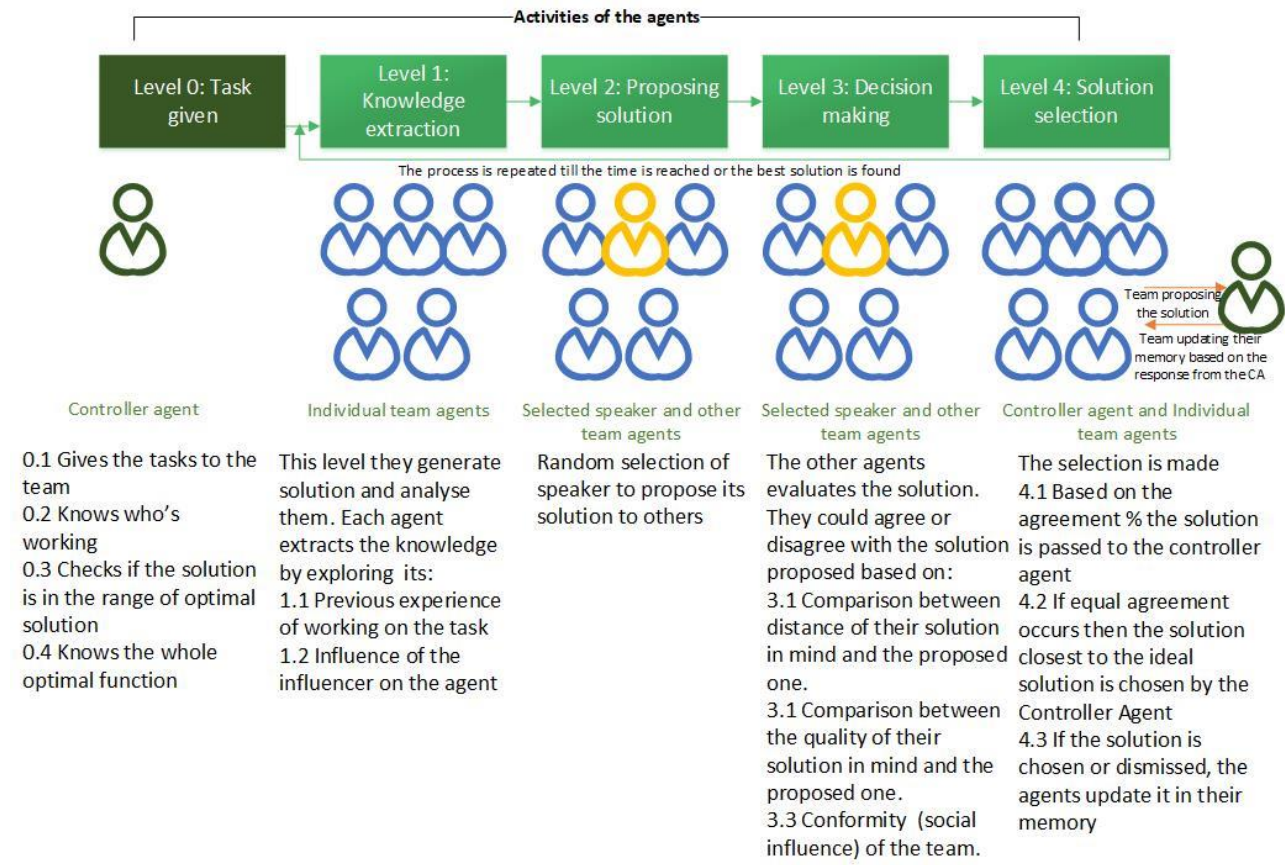

Figure 5. Model framework 
Figure 5 shows the model framework and the underlying micro-level factors that take place in a codesign process simulation. The entire episode is divided into various levels where agents take part. For example, at level 0 only the controller agent is active and gives the task (problem to be solved) to the team. At level 1, all the agents receive the task from the controller agent and generate solutions, they check whether the solution was previously proposed or not and whether it was acceptable. Then, while generating a solution they use previous experience, and/or that of the team (as each team member wants their idea to be liked/accepted by the team and therefore they may influence the agent). Similarly, at other levels, their actions and behaviours are influenced by the parameters of the model. At the end, it is the controller agent who chooses the best solution reached within the stipulated time.

Level 0: The controller agent gives a problem task to the agents, which is an objective function $f(x)$. This functions represents the $n$-dimension $(n=2$ in this case) real world problem where there are multiple possible solutions and constraints. The number of best quality solutions (depicted as light hue points in Table 1) could vary from 1 to 6 . The closer view of the solution space could be seen in the left column of Table 1 .

Level 1: Knowledge extraction is an act where an agent searches into its memory for coming up with a solution to the proposed task. For each agent, knowledge extraction depends on individual cognitive characteristics. Every agent has a different ability to explore the solution space. The way in which an agent explores the solution space is represented by the curve depicted in Figure 6. It is based on the assumption that an agent is warmed up in the initial ideation phase by triggering memory search. This is followed by the generation of new ideas (i.e., more exploration) by recalling input from its memory. However, at some point this recalling process becomes tiring, and the will to continue exploring the solution space drops towards the end of the session. By changing the $\mu$ (mean) and $\sigma$ (standard deviation) of the curve, different curves are assigned to different agents to simulate alternatives to explore the solution space. Thus, based on its cognitive style, each agent is characterized by a different way to explore and move through the solution space. Every step completed by an agent in a solution space is checked based on its past record of success or failure. Agents have different memories to either remember or forget experiences. This ability depends on: (1) time that takes to achieve success or failure and, (2) intensity of the success or failure. For example, recent best and worst solutions are more likely to be remembered by an agent than older ones. Finally, the knowledge extraction of an agent also depends on the influence of the emerging leaders/influencers members in the team. There is a probability that an agent might be attracted to a previous solution proposed by another agent more than to its own current proposed solution. The level of attraction may increase in case that the other agent has higher importance or plays a more relevant role in the team.

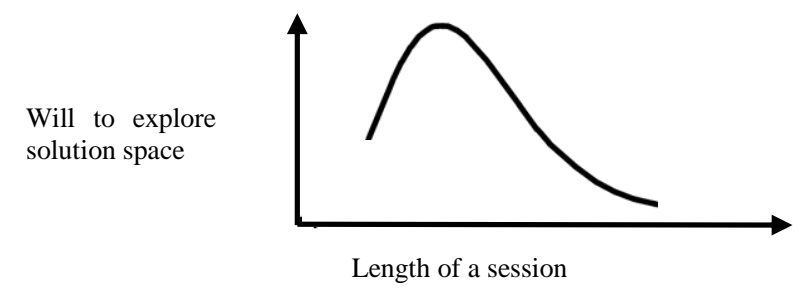

Figure 6. The way that an agent explores a solution space

Level 2: This final solution is stored in the 'minds' of all the agents. A random speaker agent is selected to communicate its solution to the other agents in the team.

Level 3: The other agents could agree or disagree with the proposed solution suggested by the speaker agent. Let s1, s3, s4 and s5 be the solutions other agents (A1, A3, A4, and A5) have in mind, and s2 be the solution proposed by the speaker agent (A2) on the solution space. Agents evaluate the proposed solution and makes decisions by following three alternative scenarios depicted in Table 1. However, the probability to satisfy or conform the group is dissimilar for some agents - i.e., there might be agents that disagree with the preferred solution of the group.

Level 4: Based on the team agreement percentage, the solution is communicated to the controller agent, who decides further whether the solution is in the acceptable range. If the agreement percentage is below certain threshold, the process is repeated again, starting from level 1. 
Table 1. Decision-making in the model

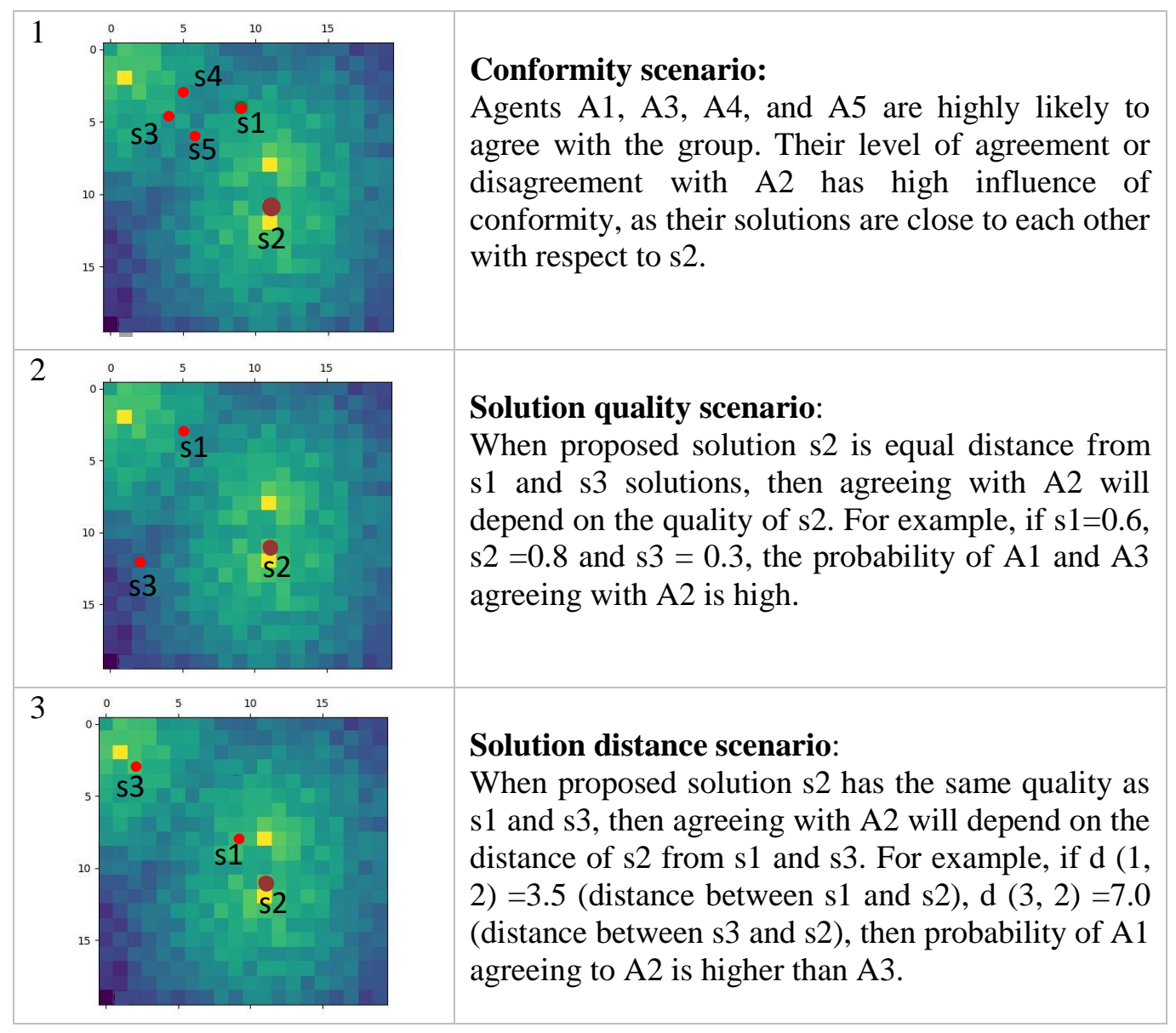

\section{CONCLUSION}

To summarise, the proposed model builds upon various psychological and design theories that form a solid ground for 'what-if' studies. The conceptual model is based on leading theories, rules, assumptions and approaches that individuals adopt while working in a co-design team. The model focuses on sociocognitive aspects of a team that were not much investigated in the past, with a focus on the interactions established among its members. The parameters of the model were classified into independent, intermediate and dependent variables. The model framework incorporates cognitive and behavioural characteristics of team members, and describes how they interact at different levels of co-design activity. The framework is characterized by independent parameters which control the co-design process like session length, session numbers and addition/removal of agents that have consequences on intermediate/dependent variables. It explores individual features like learning from experience, forgetfulness, self-efficacy and how an agent may emerge as a team influencer. Social influence (conformity) viewed as a team characteristic has to be investigated to understand its ultimate impact on the task outcome (i.e., exploration of the solution space and quality of the solution).

The validation of the proposed conceptual framework will be conducted using computational resources implemented as an ABM tool. The authors are aware that the model is far from being exhaustive, in the sense that it misses to represent several aspects of a real- scenario. Nonetheless, the model manages to capture key social and cognitive aspects of team interaction in real design practice. An advantage of using a simpler model is that it enables to easily understand outcomes that otherwise would be complex to identify and evaluate.

In the next step, the conceptual framework will be developed into a computational model that will be used to respond the research questions presented in this study. The model will help to not only gain a better understanding onto how individual and team features influence the project outcome, but will provide a gateway for tackling such issues as well. Findings may also have implications for design practice. For example, it would help project managers, sprint masters and likely roles to adopt suitable time and task management strategies aimed at contributing to the project success. 


\section{REFERENCES}

Abar, S., Theodoropoulos, G. K., Lemarinier, P. and O’Hare, G. M. (2017), “Agent Based Modelling and Simulation tools: A review of the state-of-art software”, Computer Science Review, Vol. 24, pp. 13-33. https://doi.org/10.1016/j.cosrev.2017.03.001

Badke-Schaub, P. and Frankenberger, E. (1999), “Analysis of design projects”, Design Studies, Vol. 20 No. 5, pp. 465-480. https://doi.org/10.1016/S0142-694X(99)00017-4

Carberry, A. R., Lee, H.-S. and Ohland, M. W. (2010), "Measuring Engineering Design Self-Efficacy", Journal of Engineering Education, Vol. 99 No. 1, pp. 71-79.

Carley, K. M. and Gasser, L. (1999), “Computational organization theory”. In: Multiagent Systems: A Modern Approach to Distributed Artificial Intelligence, s.l.: MIT Press, pp. 1-32.

Dionne, S. D. and Dionne, P. J. (2008), "Levels-based leadership and hierarchical group decision optimization: A simulation", The Leadership Quarterly, Vol. 19 No. 2, pp. 212-234. https://doi.org/10.1016/j.leaqua.2008.01.004

Dorst, K. and Cross, N. (2001), "Creativity in the design process: co-evolution of problem-solution", Design Studies, Vol. 22 No. 5, pp. 425-437. https://doi.org/10.1016/S0142-694X(01)00009-6

Gero, J. S. and Kannengiesser, U. (2004), "Modelling Expertise of Temporary Design Teams”, Journal of Design Research, Vol. 4, pp. 1-13.

Herrmann, J. W. (2015), "Predicting the Performance of a Design Team Using a Markov Chain Model", IEEE Transactions on Engineering Management, Vol. 62 No. 4, pp. 507-516. https://doi.org/10.1109/TEM.2015.2456833

Jamshidnezhad, B. and Carley, K. M. (2015), "Agent-based modelling of quality management effects on organizational productivity”, Journal of Simulation, Vol. 9 No. 1, pp. 73-82. https://doi.org/10.1057/jos.2014.26

Jin, Y. and Levitt, R. E. (1996), “The Virtual Design Team: A Computational Model of Project Organizations”, Computational \& Mathematical Organization Theory, Vol. 2 No. 3, pp. 171-196.

Kelman, H. C. (1958), "Compliance, identification, and internalization three processes of attitude change”, Journal of Conflict Resolution, Vol. 2 No. 1, pp. 51-60.

Leibowitz, N., Baum, B., Enden, G. and Karniel, A. (2010), “The exponential learning equation as a function of successful trials results in sigmoid performance", Journal of Mathematical Psychology, Vol. 54 No. 3, pp. 338-340. https://doi.org/10.1016/j.jmp.2010.01.006

McComb, C., Cagan, J. and Kotovsky, K. (2015), "Lifting the Veil: Drawing insights about design teams from a cognitively-inspired computational model”, Design Studies, Vol. 40, pp. 119-142. https://doi.org/10.1016/j.destud.2015.06.005

Perišić, M. M., Štorga, M. and Gero, J. S. (2019), "Exploring the Effect of Experience on Team Behavior: A Computational Approach", International Conference on - Design Computing and Cognition, Lecco, Italy, https://doi.org/10.1007/978-3-030-05363-5_32

Prussia, G. E., Anderson, J. S. and Manz, C. C. (1998), "Self-leadership and performance outcomes: the mediating influence of self-efficacy", Journal of Organizational Behavior, Vol. 19, pp. 523-538. https://doi.org/10.1002/(SICI)1099-1379(199809)19:5<523::AID-JOB860>3.0.CO;2-I

Salas, E. et al. (2005), "Modeling Team Performance: The Basic Ingredients and Research Needs”, In: Rouse W. B. and Boff K. R. (Eds.), Organizational simulation. Wiley, Hoboken, NJ, pp. 185-228. https://doi.org/10.1002/0471739448.ch7

Sayama, H., Farrell, D. L. and Dionne, S. D. (2010), “The Effects of Mental Model Formation on Group Decision Making: An Agent-Based Simulation”, Complexity, Vol. 16 No. 3, pp. 49-57. https://doi.org/10.1002/cplx.20329

Singh, V. (2009), Computational Studies on the Role of Social Learning in the Formation of Team Mental Models, Design Lab Faculty of Architecture, Design and Planning, The University of Sydney, Sydney.

Singh, V. and Casakin, H. (2015), "Developing a computational framework to study the effects of use of analogy in design on team cohesion and team collaboration", International Conference on Engineering Design, ICED15, Milan, Italy

Singh, V., Dong, A. and Gero, J. S. (2011), “How important is team structure to team performance?”, International Conference on Engineering Design, ICED11, Technical University of Denmark.

Sosa, R. and Gero, J. S. (2013), "The creative value of bad ideas: A computational model of creative ideation", The Association for Computer-Aided Architectural Design Research in Asia (CAADRIA), Hong Kong, and Center for Advanced Studies in Architecture (CASA), Department of Architecture-NUS, Singapore, pp. 853-862

Sparks, J. (2018), Types of Conformity. [Online]. Available at: https://www.tutor2u.net/psychology/reference/typesof-conformity

Stempfle, J. and Badke-Schaub, P. (2002), "Thinking in design teams - an analysis of team communication", Design Studies, Vol. 23 No. 5, pp. 473-496. https://doi.org/10.1016/S0142-694X(02)00004-2 